\title{
Evaluación de cicatrización en zonas donantes de injerto de piel parcial con uso de xenoinjerto en comparación con sustituto dérmico sintético de celulosa
}

\author{
Enrique Antonio Chau Ramos*1,a,b
}

RESUMEN

Objetivo: Realizar una evaluación de la cicatrización en la zona donante de injerto de piel parcial con uso de sustituto dérmico comparado con xenoinjerto, en pacientes con lesiones diversas que requirieron injerto de piel parcial. Materiales y métodos: Se presenta el reporte de 20 pacientes entre 19 y 65 años de la Unidad de Cirugía Plástica del Hospital María Auxiliadora de Lima Metropolitana-Perú, entre diciembre 2017 y junio 2018, donde se evaluó la cicatrización en zonas donantes de injerto de piel parcial. El estudio es de tipo intervención, analítico, prospectivo y longitudinal. Emplea el diseño doble ciego para controlar posibles sesgos. Para analizar la significancia estadística se usaron pruebas no paramétricas con un nivel de confianza $95 \%$.

Resultados: Con el uso del sustituto dérmico se aprecia una mejor calidad de cicatrización de zonas donantes de epitelización en comparación con el xenoinjerto. Ambas técnicas se evaluaron con la escala de Vancouver que considera cinco aspectos (cicatrización, vascularidad, pigmentación, flexibilidad y altura), de los cuales, la cicatrización tuvo resultados significativos $(\mathrm{p}<0,05)$. Al estimar el riesgo de evolución de cicatrización según el modelo de riesgos proporcionales de Cox, se obtuvo un $\mathrm{H}=0,60$ (IC95\% 0,46-0,78), lo cual indica que el menor tiempo de cicatrización se encontró en el grupo en que se empleó el sustituto dérmico.

Conclusiones: El sustituto dérmico es una alternativa importante que favorece buena calidad de la cicatrización en las zonas donantes. El sustituto dérmico es más eficiente que el xenoinjerto convencional al ser evaluado y comparado en la escala de cicatrización de Vancouver.

Palabras clave: Injerto; Xenoinjertos; Tejido de granulación; Cicatrización (Fuente: DeCS BIREME).

\section{Evaluation of healing in partial skin graft donor sites using a xenograft compared to a synthetic cellulose skin substitute}

\section{ABSTRACT}

Objective: To evaluate healing in partial skin graft donor sites using a skin substitute compared to a xenograft in patients with different diseases requiring partial skin grafting.

Materials and methods: This paper presents a report of 20 patients between 19 and 65 years from the Plastic Surgery Unit of the Hospital María Auxiliadora in Lima Metropolitan Area, Peru, between December 2017 and June 2018, where healing was evaluated in partial skin graft donor sites. An interventional, analytical, prospective and longitudinal study was conducted using a double-blind design to control possible biases. For the statistical significance analysis, nonparametric tests with a $95 \%$ confidence interval were used.

Results: Using a skin substitute, a better healing quality of donor sites of epithelialization was seen compared with xenografting. Both techniques were evaluated with the Vancouver scale, which considers five aspects (healing, vascularity, pigmentation, flexibility and height), out of which healing showed significant results $(\mathrm{p}<0.05)$. Estimation of the risk in the healing process according to the Cox proportional hazards model showed that $\mathrm{H}=0.60(95 \% \mathrm{Cl} 0.46$ 0.78), which indicates that the shortest healing time was found in the skin substitute group.

Conclusions: Skin substitutes are an important alternative that favors the good quality of healing in donor sites. skin substitutes proved to be more effective than conventional xenografting when evaluated and compared using the Vancouver healing scale.

Keywords: Transplantation; Heterografts; Granulation tissue; Wound healing (Source: MeSH NLM).

1. Hospital María Auxiliadora, Unidad de Cirugía Plástica. Lima, Perú.

a. Magister en Cirugía Plástica y Reconstructiva.

b. Miembro de la Sociedad Peruana de Cirugía Plástica Reconstructiva.

*Autor corresponsal 


\section{INTRODUCCIÓN}

Actualmente, ocurren accidentes que ocasionan diversas lesiones y, en varios casos, es necesario el uso de injerto de piel parcial para cobertura, como parte de una técnica reconstructiva. Existen diversos substitutos dérmicos que han sido estudiados por diversos autores que han intentado encontrar el reemplazo dérmico ideal según las necesidades y complicaciones del paciente ${ }^{(1)}$.

Los injertos representan un gran desafío para los cirujanos plásticos debido a los resultados de cicatrización de las zonas dadoras. Los avances realizados han desarrollado diferentes técnicas y productos para cobertura temporal de la zona donante como apósitos biológicos o sintéticos y, además, se han obtenido diversos resultados en la cicatrización de estas zonas. Es por ello que el correcto uso de los apósitos sintéticos permite aislar, proteger y optimizar el proceso de cicatrización, si ha sido elegido adecuadamente, y brinda un ambiente óptimo que preserve los principios fisiológicos básicos de humedad, calor, circulación sanguínea y oxigenación que son necesarios para la cicatrización (2).

El proceso normal de cicatrización dura aproximadamente 6 meses. En las fases iniciales, es importante el uso de apósitos de diferentes tipos como xenoinjertos, apósitos sintéticos, etc., en la zona donadora de piel parcial. El objetivo es encontrar el que brinde mayor beneficio en la calidad de cicatrización y que facilite una adecuada recuperación de la zona donante en los pacientes afectados ${ }^{(3)}$.

En el Hospital María Auxiliadora se presentan, aproximadamente, 1200 casos anuales que requieren de injerto de piel, en los que se utilizan diferentes métodos para protección o cobertura temporal de la zona donante; entre ellos, apósitos sintéticos o biológicos, según la disponibilidad. Son notorias las diferencias que existen en el proceso de cicatrización con los usos de los diferentes apósitos.

Debemos destacar que, en los últimos años, la cirugía plástica ha desarrollado más innovaciones para mejorar las técnicas de injerto, lo cual es importante para el presente estudio, que busca nuevas alternativas de cicatrización con apósitos biológicos o sintéticos.

\section{MATERIALES Y MÉTODOS}

\section{Población de estudio}

Se presenta el reporte de 20 pacientes entre 19 a 65 años de la Unidad de Cirugía Plástica del Hospital María Auxiliadora de Lima Metropolitana-Perú, entre diciembre 2017 y junio 2018. Se realizó un muestreo no probabilístico. No se trabajó con muestra, ya que se consideró el total de pacientes que utilizaron las dos técnicas.

Diseño de estudio

El estudio es de tipo intervención, analítico, prospectivo y longitudinal. Se realizó una toma de injerto de piel parcial con dermatomo Humeca calibrado a 0,08 mm; se empleó una solución vasoconstrictora de 500 cc de cloruro de sodio, 20 cc de xilocaína en $2 \%$, y 1 cc de epinefrina de $1 \mathrm{~m}$. Se infiltró en el tejido celular subcutáneo con aguja $\mathrm{n} .{ }^{\circ} 21$ de 2 pulgadas y media de longitud en zona delimitada previamente.

El xenoinjerto y el sustituto dérmico se aplicaron en diferentes zonas anatómicas, ubicadas en lados contrarios y separadas por un puente de piel indemne que sobrepasaba $1 \mathrm{~cm}$ los bordes de la zona donante. Se tomó en cuenta una posible movilización del xenoinjerto o sustituto dérmico, bajo el modelo de diseño pareado.

La importancia de este estudio es evaluar la calidad de la cicatrización de ambas zonas donantes, una con xenoinjerto y la otra con sustituto dérmico, en un periodo de tres meses y, a través de ello, diferenciar cuál de los dos procedimientos es una alternativa con potencial beneficio de mejores resultados en el proceso de cicatrización de la zona donante ${ }^{(3,4)}$.

El sustituto dérmico es un apósito fabricado con un biomaterial hecho de celulosa generada biotecnológicamente a base de azúcar. Tiene un contenido de agua de al menos $95 \%$ y un pH de 7. Proporciona agua a la piel y permite un largo tiempo sin deshidratación. Este producto tiene múltiples ventajas, entre ellas, proporciona un ambiente húmedo que promueve la mejora del equilibrio hídrico y la permeabilidad al vapor de agua. Además, es permeable para el aire y líquidos, suave y semitransparente. La superficie lisa e hidratada asegura una eliminación sin dolor y no se adhiere a la herida, proporciona protección contra la invasión de patógenos y tiene una excelente biocompatibilidad ${ }^{(3-5)}$.

Para evaluar los resultados en la cicatrización de las dos zonas donantes cubiertas con xenoinjerto y el sustituto dérmico respectivamente, se utilizó la escala de Vancouver en la que se consideran los parámetros de pigmentación, vascularidad, flexibilidad y altura ${ }^{(3)}$.

Para realizar el procedimiento se toman dos zonas de piel del mismo paciente con iguales dimensiones $(6 \times 10 \mathrm{~cm})$. La zona donante $A$ se cubrió con sustituto dérmico y la zona $B$, con el xenoinjerto liofilizado. 
La toma de piel se realiza con el uso de dermatomo calibrado y manipulado por el mismo operador. Este instrumento sirve para obtener injertos de piel de espesor y anchura variable. Está diseñado e indicado para utilizarse en el corte de láminas de piel y luego emplearla como injerto en cirugía plástica ${ }^{(5,6)}$.

\section{Variables y mediciones}

La medición de la cicatrización se realiza con la escala Vancouver (Escala de valoración objetiva paciente, observador). Es el instrumento de medición de cicatrización más reconocida que evalúa 4 variables: vascularización, altura, flexibilidad y pigmentación. El evaluador mide la cicatriz según su percepción ${ }^{(7,8)}$.

La regresión de Cox es una clase de modelo usados para estimar los riesgos que afectan a la supervivencia de una población. En este estudio se empleó este procedimiento para estimar el riesgo de evolución de la cicatrización.

Los criterios de exclusión fueron no requerir injertos de piel parcial o causas múltiples, no tener consentimiento informado de la aplicación del sistema, alteración de la zona donante, presencia de comorbilidades, pacientes con problemas psicológicos o psiquiátricos, o que no colaboren con el tratamiento y gestantes ${ }^{(9,10)}$.

Según las características evaluadas de epitelización, en

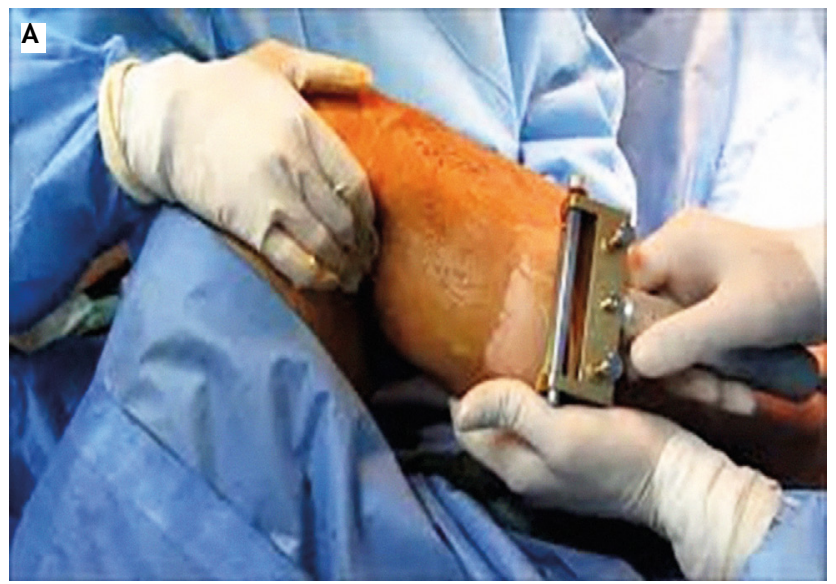

Figura 1. Extracción de dos zonas de piel parcial

La zona donante A se cubrió con sustituto sintético de piel, mientras que en la zona $B$ se colocó un xenoinjerto (Figuras 2A- 2B). Después la zona se cubrió con gasa parafinada más gasa apósito. Se realizaron cambios ambos casos se aprecia mejores resultados con el sustituto dérmico, por presentar una zona donante con mejores características que la zona con uso de xenoinjerto.

\section{Consideraciones éticas}

El trabajo de investigación cumplió con los aspectos éticos de beneficencia y justicia. Se respetó la confidencialidad de los pacientes que, a través de un consentimiento informado, autorizaron y confirmaron su participación antes de la intervención. Los aspectos éticos están relacionados en mantener confidencialidad con la información de datos y fotografías obtenidas en el estudio y el anonimato del paciente. La investigación tiene los permisos del Comité de Ética del Hospital y el permiso del director y jefe de servicio de Cirugía Plástica de la Clínica.

\section{RESULTADOS}

El procedimiento se realizó en la Unidad de Cirugía Plástica del Hospital María Auxiliadora. Se extrajeron dos tomas de piel de dos zonas separadas por un puente de piel total (zona A y zona B). Ambas miden $0,8 \mathrm{~mm}$ de grosor y 2 pulgadas de ancho. Antes de la extracción, las zonas donantes se infiltraron con solución de xilocaína al $2 \%$ más epinefrina $1 / 40000$ y luego se realizó la toma con un dermatomo calibrado. Las extracciones fueron realizadas por el mismo cirujano (Figuras 1A y 1B).

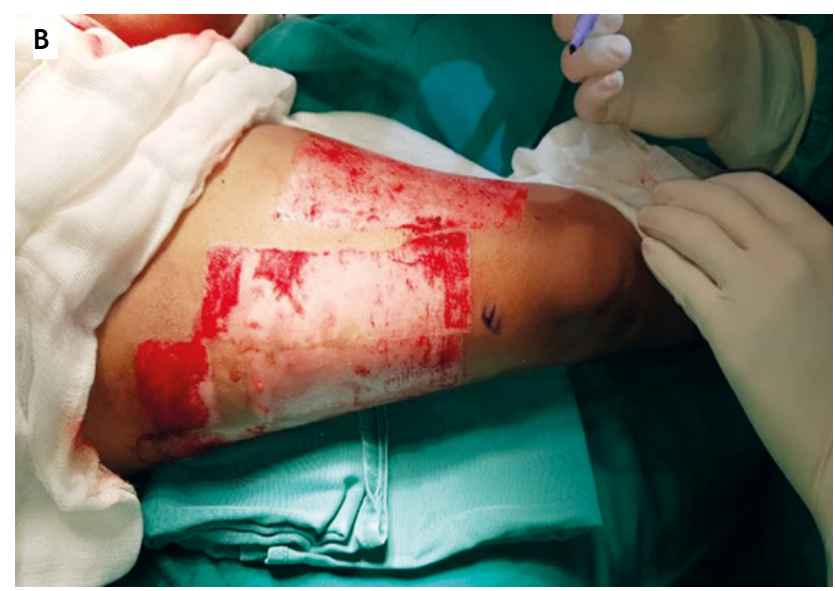

y revisiones semanales hasta que se evidenció la epitelización en la zona donante. La cicatrización se evaluó en el tercer mes de evolución. 

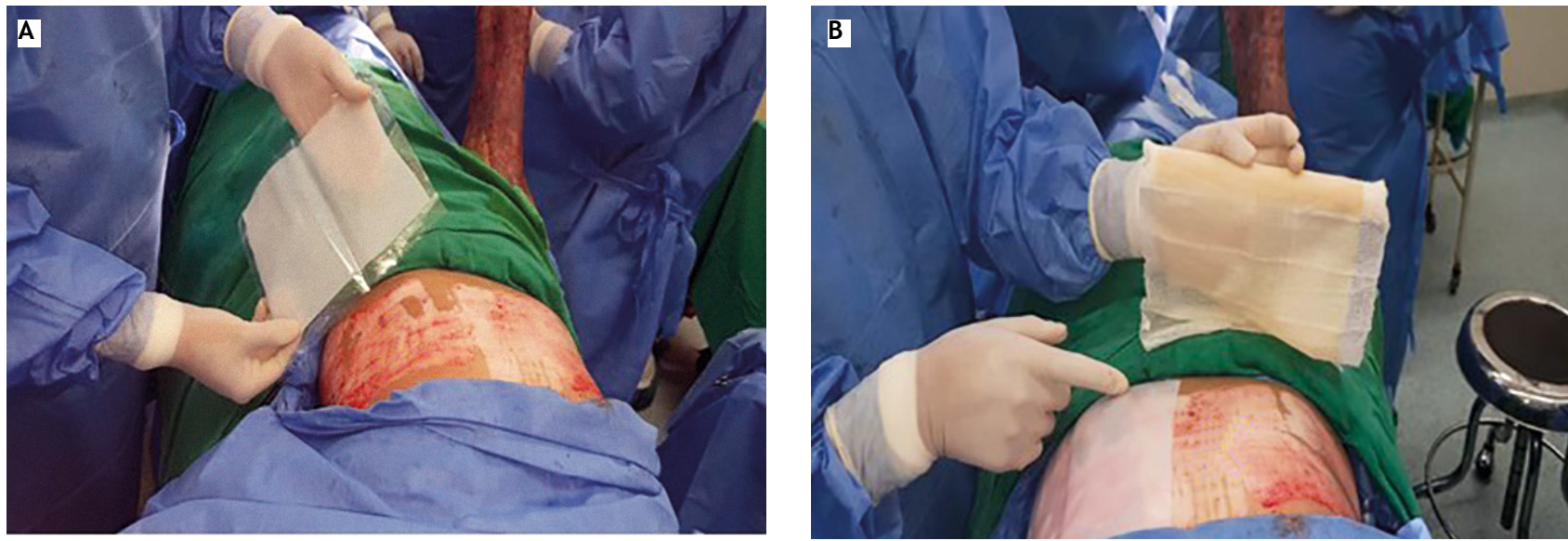

Figura 2. Colocación de xenoinjerto y sustituto dérmico en las zonas donantes

Para evaluar las dos técnicas y medir la calidad de la cicatrización en el tercer mes, se empleó la escala Vancouver que se basa en los parámetros de vascularización, altura, flexibilidad y pigmentación. La importancia del estudio es comparar los dos apósitos $y$, a través de ello diferenciar que sustituto de piel es una mejor alternativa; con potencial beneficio de recuperación rápida y efectiva (Figura 3).

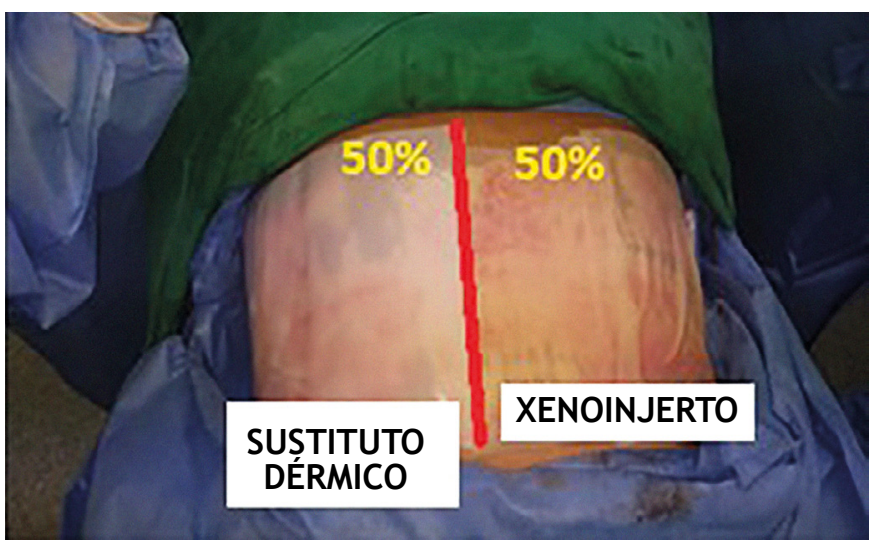

Figura 3. Aplicación de las dos técnicas (sustituto dérmico y xenoinjerto)

El sustituto dérmico presenta mejores resultados que el xenoinjerto convencional, al ser evaluado y comparado en la escala de cicatrización Vancouver (VSS) que es reconocida para la calibración de cicatrices, y que cuenta con 4 variables (vascularidad, pigmentación, altura y flexibilidad). Se observan cambios estadísticamente significativos en la cicatrización, que favorecen a la técnica del sustituto dérmico $(p<0,05)$.

En la figura 4 se pueden observar los resultados de las dos técnicas según porcentajes significativos. La pigmentación de la piel con la técnica del sustituto dérmico tuvo mejores resultados $(0,30)$ que el xenoinjerto $(1,10)$, lo mismo ocurre con la vascularidad (sustituto dérmico, 0,55 y xenoinjerto, 1,55). La flexibilidad de la piel (en sus características de resistencia, flexibilidad y elasticidad) obtuvo un valor de 0,15 con el apósito sintético y 1,25 con el xenoinjerto. La altura obtiene un mejor resultado $(0,95)$ con sustituto dérmico que con el xenoinjerto $(1,50)$. La comparación final de las dos técnicas en cicatrización según la escala de Vancouver, muestra un resultado más satisfactorio con el empleo del sustituto dérmico $(1,95)$ frente al uso de xenoinjerto $(5,40)$. 


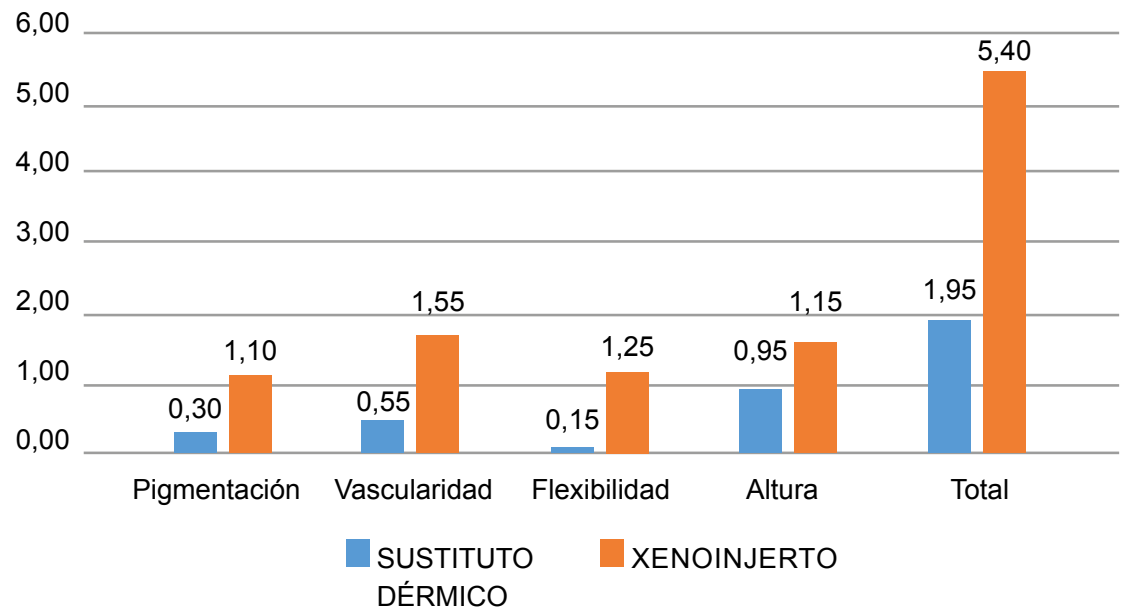

Figura 4. Comparación de la efectividad de sustituto dérmico versus xenoinjerto

Se conoce como regresión de Cox a una clase de parámetros usados para modelar los riesgos que afecta a la supervivencia de una población ${ }^{(11)}$. Al estimar el riesgo de evolución de cicatrización según el modelo de riesgos proporcionales de $\mathrm{Cox}$, se obtuvo un $\mathrm{H}=0,60$ (IC95 \% 0,46-0,78), lo cual indica que el menor tiempo de cicatrización se encontró en el grupo en que se empleó el sustituto dérmico (Tabla 1).

Tabla 1. Análisis de regresión de Cox

\begin{tabular}{|c|c|c|c|c|c|c|c|c|}
\hline \multicolumn{9}{|c|}{ Variables en la ecuación - Riesgo de evolución de cicatrización } \\
\hline & \multirow[t]{2}{*}{ B } & \multirow[t]{2}{*}{ SE } & \multirow[t]{2}{*}{ Wald } & \multirow[t]{2}{*}{ df } & \multirow[t]{2}{*}{$\mathbf{p}$} & \multirow[t]{2}{*}{$\mathbf{H}$} & \multicolumn{2}{|c|}{$95,0 \% \mathrm{Cl}$ for $\mathrm{H}$} \\
\hline & & & & & & & Lower & Upper \\
\hline Calidad & -.505 & .134 & 14,262 & 1 & .000 & .603 & .464 & .784 \\
\hline
\end{tabular}

Chi-cuadrado $=18,49 \mathrm{p}=0,000$

\section{DISCUSIÓN}

En 2009, Quezada realizó una investigación sobre el empleo de sustitutos dérmicos en secuelas de quemaduras, en sus variables de cicatrización, elasticidad y plegabilidad. A los dos años la piel tenía aspecto similar, sin fibrosis ni contracción del injerto. El excelente resultado estético-funcional, con plegabilidad y elasticidad, constituye una alternativa real de tratamiento para algunos pacientes con secuelas de quemaduras y es posible su uso en forma ambulatoria sin mayores complicaciones. Nuestro estudio de comparación de apósitos entre xenoinjerto y el sustituto dérmico resalta la importancia de buscar nuevas y mejores alternativas de cicatrización con apósitos biológicos o sintéticos ${ }^{(12)}$.

Urbina $G$, en un estudio de 2016, empleó sustitutos sintéticos dérmicos en el manejo de heridas complejas como una posibilidad de obtener una cicatriz estética y funcionalmente adecuada. En nuestra investigación se evalúan los resultados del empleo del sustituto dérmico con la escala de Vancouver mediante la comparación con el xenoinjerto; obtuvimos un resultado óptimo con el empleo del sustituto dérmico. Nuestro trabajo contribuye a ampliar el conocimiento sobre estos 
sustitutos en zonas donantes, sus beneficios y las posibilidades de disminuir los riesgos potenciales en la etapa de cicatrización ${ }^{(13)}$.

Estudiamos los beneficios del sustituto dérmico como nueva alternativa en el cuidado de la zona donante de injerto de piel parcial. El apósito de gel se utiliza para cubrir las zonas donantes y brindar un medio adecuado que propicie una cicatrización en mejores condiciones. Para conocer los resultados en la cicatrización de las dos zonas donantes cubiertas con xenoinjerto y el sustituto dérmico se utilizó la escala de Vancouver con los diferentes parámetros como pigmentación, vascularidad, flexibilidad y altura ${ }^{(14,15)}$.

Se aprecia mejor calidad en la cicatrización con el sustituto dérmico, en comparación con el xenoinjerto, en la mayoría de las variables analizadas en la escala de Vancouver. Según los resultados encontrados, la diferencia en la calidad en la cicatrización de la zona donante con uso del sustituto dérmico es estadísticamente significativa comparado con el uso de xenoinjerto $(p<0,05)^{(12,13)}$.

En conclusión, este estudio confirma que el sustituto dérmico es una nueva alternativa para la cicatrización de la zona donante respecto a xenoinjerto como se da en otros apósitos.

Se deben ampliar las investigaciones que impliquen, estudios comparativos con el sustituto dérmico y las alternativas vigentes, y desarrollar metaanálisis con otras experiencias internacionales con la finalidad de aumentar el tamaño de muestra y analizar la efectividad del apósito, complementando los resultados con evaluación histológica para reforzar la efectividad del sustituto dérmico en cicatrización y ampliar nuevas investigaciones científicas para antecedentes de diversos estudios ${ }^{(13,16)}$.

Se recomienda realizar nuevos estudios que comparen la calidad de cicatrización del sustituto dérmico con diferentes apósitos y su uso en el manejo de diferentes tipos de lesiones.

\section{REFERENCIAS BIBLIOGRÁFICAS}

1. Rodríguez Villalonga L, Chirino Díaz L, García Pelegrí S, Borrás Migues M, Quiñones Castro M, Durán González S, et al. Fototerapia en el tratamiento de las zonas donantes del cuerpo humano para los injertos libres de piel. Rev Cubana Angiol Cir Vasc. 2008; 9(1): 1-9.

2. Mora-González R, Hernández-López AE, Polo-Soto SM. Estudio comparativo experimental entre xenoinjerto de dermis acelular humana desnaturalizada y xenoinjerto de esclera porcina desnaturalizada para evaluar la integración del injerto en defectos esclerales de espesor parcial. Rev Sanid Milit Méx. 2004; 58(2): 59-64.

3. ORSKIN-EPICITE: next generation wound dressing. [Internet]. 2018. Disponible en: https://www.qrskin.com/ epicite-hydro-for-skin-graft-surgery/properties.html

4. López Delis A, Rodrigues Fleury Rosa SS, Narcizo de Souza PE, Carneiro MLB, Fleury Rosa MF, Lobo Macedo YC, et al. Characterization of the cicatrization process in diabetic foot ulcers based on the production of reactive oxygen species. J Diabetes Res. 2018; 1: 1-10.

5. Koudoukpo C, Atadokpèdé $F$, Abègbidi H, Assogba $F$, Akpadjan F, Dégboé B, et al. Évaluation clinique du délai de cicatrisation des lésions d'ulcère de Buruli de diamètre inférieur ou égal à 10 centimètres à pobè (Bénin). Ann Dermatol Venereol. 2015 Dec; 142(12): S612.

6. Toniollo CL, Da Matta ES. Abordagem multidisciplinar na cicatrização de úlcera venosa crônica. BJSCR. 2015; 11(3): 12-6.

7. Vásquez GD, Fierro AL, Arellano MI, Tirado SA, Peniche Castellanos A. Estudio comparativo entre el uso de apósito hidrocoloide vs uso de tie-over para valorar el porcentaje de integración de los injertos cutáneos de espesor total. Dermatología Rev Mex. 2011; 55(4): 175-9.

8. Rapado Raneque M, Rodríguez Rodríguez A, Penich Covas C. Hydrogel wound dressing preparation at the laboratory scale by using electron beam and gamma radiation. Nucleus. 2013; 53: 24-31.

9. Casierra-Posada F, Guzmán JA. Efecto del portainjerto y del injerto intermedio sobre la calidad de fruta en mango (Mangifera indica L). Agron Colomb. 2009; 27(3): 367-74.

10. Paredes E, Castillo Fernández L, Gómez Beltrán O, Vargas Cruz V, Lasso Betancor CH, Granero Cendó R, et al. Nuestra experiencia en el manejo de quemaduras con apósito antimicrobiano de plata, carbón activo y tecnología Safetac ${ }^{\circledR}$. Acta Pediatr Esp. 2013; 71(8): 165-71.

11. Zamora J. Regresión de Cox. Unidad de Bioestadística Clínica - IRYCIS [Internet]. 2013. Disponible en: ftp://ftp. hrc.es/pub/bioest/charlas/Analisis_de_Supervivencia_3. pdf

12. Quezada B, Ayala R, Yanez V. Uso de sustituto dérmico en niños con secuelas de quemaduras: tres casos clínicos. Rev Chil Pediatr. 2008; 80(2): 150-6.

13. Urbina G, Rider J. Manejo de heridas complejas con sustitutos dérmicos. Rev Chil Cir. 2016; 68(3): 245-9.

14. $\mathrm{KCl}$ Medical. V.A.C. Freedom $®$ : El sistema portátil para la curación eficaz de heridas [Internet]. 2016. Disponible en: https://www.acelity.com/

15. Salem C, Pérez JA, Henning E, Uherek F, Schultz C, Butte JM, et al. Heridas: Conceptos generales. Cuad Cir. 2000; 14(3): 90-9.

16. Sosa-Serrano AFJ, Álvarez-Díaz CJ, Cuenca-Pardo J, JuárezAguilar E, Kuri-Harcuc W. Tratamiento de quemaduras de espesor total mediante autoinjertos mallados cubiertos con aloinjertos criopreservados de epidermis humana cultivada in vitro. Reporte de un caso. Cir Plast. 1999; 9(3): 126-9. 
Evaluación de cicatrización en zonas donantes de injerto de piel parcial con uso de xenoinjerto en comparación con sustituto dérmico sintético de celulosa

Fuentes de financiamiento:

Este artículo ha sido financiado por el autor.

Conflictos de interés:

El autor declara no tener ningún conflicto de interés.

Correspondencia:

Enrique Antonio Chau Ramos

Dirección: Av. Javier Prado Oeste1351, dpto.1304, San Isidro.

Lima, Perú.

Teléfono: (+51) 948029959

Correo electrónico: drenriquechau@gmail.com
Recibido: 19 de setiembre de 2018.

Evaluado: 07 de diciembre de 2018

Aprobado: 11 de febrero de 2019

(c) La revista. Publicado por Universidad de San Martín de Porres, Perú. (c) $\mathbf{\text { вr }}$ Licencia de Creative Commons Artículo en acceso abierto bajo términos de Licencia Creative Commons Atribución 4.0 Internacional. (http://creativecommons.org/licenses/by/4.0/)

ORCID iDs

Enrique Antonio Chau Ramos

https://orcid.org/0000-0003-1482-2614 